\title{
VASCULAR PLANT DIVERSITY IN THE SACRED GROVE OF MODAPALLI IN VISKHAPATNAM DISTRICT OF ANDHRA PRADESH, INDIA
}

\author{
M. Tarakeswara Naidu ${ }^{1}$, O. Aniel Kumar ${ }^{2} \&$ M. Venkaiah ${ }^{3}$ \\ 1,2,3 Department of Botany, Andhra University, Visakhapatnam, Andhra Pradesh 530003, India \\ ${ }^{1}$ tarakeswaranaidu@gmail.com (corresponding author), ${ }^{2}$ oak.aniel@gmail.com, ${ }^{3}$ venkaiah_botany@rediffmail.com
}

ISSN 0974-7907 (Online) ISSN 0974-7893 (Print)

Abstract: Sacred groves are tracts of virgin forest with rich biodiversity, as they have been protected for centuries by the local people for their cultural and religious beliefs and taboos. Sacred groves are representatives of climax vegetation and exhibit diversity of species such as trees, climbers and other shade loving herbs. In the present investigation, an attempt was made to study the diversity of a sacred grove of Modapalli, Visakhapatnam District, which comes under the Eastern Ghats of India. A total of 207 species, 181 genera and 78 families comprising pteridophytes, gymnosperms and angiosperms were reported in the sacred grove. In this study, only one endemic species, i.e., Ophiorrhiza chandrasekharanii and a few threatened species were recorded.

Keywords: Conservation, Modapalli, plant diversity, sacred grove, threatened taxa.

India has a long tradition of prudent use and wise conservation of all resources that are useful to people. Traditionally, the local people have been preserving small patches of relatively dense forests based on religious values and beliefs. These are called 'sacred groves' and act as treasure houses for large numbers of endemic and rare plants of the region (Chandrashekara \& Sankar 1998; Jamir \& Pandey 2003; Sujana \& Sivaperuman 2008). Sacred groves thus are the relics of vegetation which have survived under a variety of ecological situations in India and are the present hot spots of biodiversity (Rao 1996). In the tropics, sacred groves play a vital role in traditional biodiversity conservation and improve the soil stability of the area and prevent soil erosion too (Rawat et al. 2011). This way of conserving natural biodiversity through preservation plots in forest areas or sacred groves are a unique feature in Indian culture (Khullar 1992). Sacred groves are true indicators of the types of vegetations that once existed before the dawn of modern civilization and their existence is due to certain taboos, strong beliefs and supplemented mystic folklore (Gadgil \& Varthak 1975). In the recent past, due to change of socio-economic conditions and land use systems, many sacred groves are threatened and altered, both in terms of size, vegetation structure
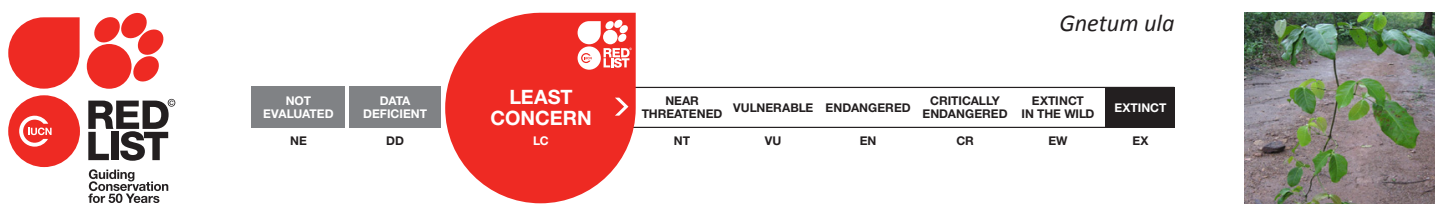

DOI: http://dx.doi.org/10.11609/JoTT.03550.7683-90

Editor: B. Ravi Prasad Rao, Sri Krishnadevaraya University, Anantapuramu, India.

Date of publication: 26 August 2015 (online \& print)

Manuscript details: Ms \# 03550 | Received 05 March 2013 | Final received 20 July 2015 | Finally accepted 30 July 2015

Citation: Naidu, M.T, O.A. Kumar \& M. Venkaiah (2015). Vascular plant diversity in the sacred grove of Modapalli in Viskhapatnam District of Andhra Pradesh, India. Journal of Threatened Taxa 7(10): 7683-7690; http://dx.doi.org/10.11609/JoTT.03550.7683-90

Copyright: (C) Naidu et al. 2015. Creative Commons Attribution 4.0 International License. JoTT allows unrestricted use of this article in any medium, reproduction and distribution by providing adequate credit to the authors and the source of publication.

Funding: Andhra University [No U5/RF/Botany/2009].

Competing interests: The authors declare no competing interests.

Acknowledgements: The authors are grateful to the Head, Department of Botany, Andhra University for encouragement. The JoTT review process significantly improved manuscript. 


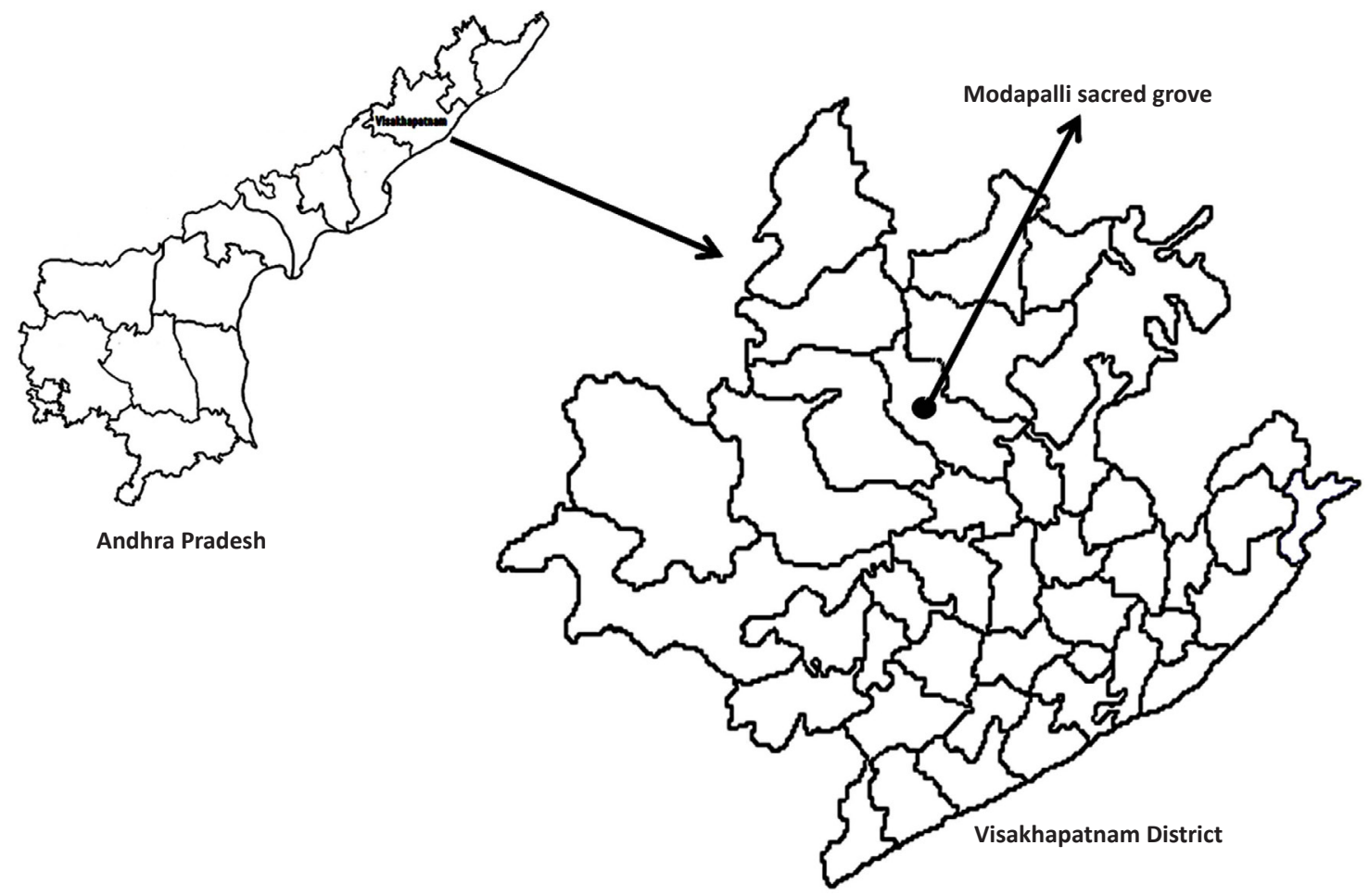

Figure 1. Map showing relative location of the Modapalli sacred grove.

and species composition. There are a number of sacred groves all over India. The erstwhile state of Andhra Pradesh, alone, has over 800 sacred groves (Rao 1996; Sunitha \& Rao 1999) and Visakhapatnam District has 31 sacred groves (Lakshminarayana \& Venkaiah 1998; Rao et al. 2010), locally known as Pavithravanalu (Rao et al. 2011).

Modapalli sacred grove is located at $18^{\circ} 02^{\prime} 324^{\prime \prime} \mathrm{N}$ \& $82^{\circ} 42^{\prime} 324^{\prime \prime} E$ in Visakhapatnam District, Andhra Pradesh (Fig. 1); it is a part of the Eastern Ghats at an altitude of $984 \mathrm{~m}$. The sacred grove extends over a 4ha area covered with mixed moist deciduous forest (Champion \& Seth 1968) which contains black alluvial soil and a perennial stream. There is a small temple of Goddess 'Modakondamma' in the middle of the sacred grove worshiped by the Bagata tribal community based on their indigenous cultural and religious beliefs and taboos. Every year, in summer they celebrate the festival of Goddess Modakondamma for three days. This captivating site of the Modakondamma temple is an alluring attraction for piligrims, who visit this place from all over Visakhapatnam District. This paper deals with vascular plant diversity in the Modapalli sacred grove with observations on threatened and endemic plant species.

\section{MATERIALS AND MethodS}

Intensive field surveys were carried out during 2010-2011, covering pre-monsoon, monsoon and post-monsoon seasons. quadruplicate specimens of each species of flowering and non-flowering vascular plants, were collected along with necessary field data. Collected specimens were made into herbarium as per the methods suggested by Jain \& Rao (1977). The collected specimens ware identified only after a critical examination with the help of different floras like Flora of the Presidency of the Madras (Gamble \& Fischer 1915-1936), Flora of Visakhapatnam District (Rao \& Kumari 2002-2008), and Flora of Vizianagaram District (Venkaiah 2004). Lists of threatened and endemic plants found in the sacred grove were prepared with the help of published works (Rao et al. 2003; Jadhav \& Reddy 2006; Reddy \& Reddy 2008). The voucher specimens were deposited at the Botany Department Herbarium (BDH), Andhra University, Visakhapatnam. The plant species status was determined based on IUCN (2014) category.

\section{RESULTS AND DISCUSSION}

Sacred groves are very important in upholding traditions and beliefs in order to protect and conserve unique forest patches which represent the relict vegetation of the concerned area. Sacred groves have 
presently attracted the attention of environmentalists, geneticists, botanists for their undisturbed natural conditions, which has made them repositories of valuable germplasm of medicinal plants, and endangered and endemic plant species (Anthwal et al. 2006).

A total of 207 species belonging to 181 genera under 78 families (Table 1 ) were reported. This is $17.2 \%$ of the species described in the flora of Visakhapatnam District (Rao \& Kumari 2002-2008) and 8\% of the flora of the state (Pullaiah \& Kuruppusamy 2008). About 95\% of the total vascular plants in the grove were angiosperms, followed by pteridophytes and gymnosperms. The genus and species ratio for the present study is $1: 1.14$, while this ratio is $1: 1.72$ for the flora of Visakhapatnam District (Rao \& Kumari 2002-2008), 1:2.86 for the flora of Andhra Pradesh (Pullaiah \& Kuruppusamy 2008) and 1:7 for the Indian sub continent (Hooker 1872-1897). Out of 207 species, nine species (4.3\%), eight genera (3.8\%) and seven families (3.3\%) are pteridophytes; one species gymnosperm i.e., Gnetum ula (0.48\%) and 197 species, 172 genera and 70 families belong to angiosperms. Among the angiosperms, there were 56 families $(71.8 \%)$ of dicotyledons and 14 families (17.9\%) of monocotyledons. The families of pteridophytes and gymnosperms were represented by seven families (9\%) and one family, i.e., Gnetaceae (1.2\%) respectively. Within the 197 angiosperms, 49 monocotyledon species (21.7\%) under 41 genera $(22.6 \%)$ were reported as compared to 152 (73.4\%) dicotyledon species under 131 genera (72.3\%). This observation is in conformity with the earlier reports (Rao \& Kumari 2002-2008; Pullaiah \& Kuruppusamy 2008) that there is predominance of dicotyledons $58 \%, 74 \%$ in the flora of Visakhapatnam District and the flora of Andhra Pradesh. The ratio of monocots to dicots among species, genera and families is $1: 3.37,1: 3$ and 1:4 respectively. Habitat wise analysis of flora shows comparatively higher percentage of herbs $(47.8 \%)$ followed by trees $(28.5 \%)$, climbers $(12.5 \%)$ and Shrubs (11.1\%).

The dominant family is Euphorbiaceae represented by 15 species, followed by Poaceae (11), Orchidaceae and Rubiacae (with 10 species each), Asteraceae (8 species), Fabaceae (7 species), and Araceae, Moraceae, Rutaceae and Verbenaceae (with 6 species each), Acanthaceae, Commelinaceae, Lamiaceae and Urticaceae (with 5 species each), Meliaceae and Piperaceae (with 4 species each), Adiantaceae, Dioscoreaceae, Malveceae, Mimosaceae, Ranunculaceae, Solanaceae and Zingeberaceae (with 3 species each), 19 families have two species each while 37 families were represented by just a single species. Euphorbiaceae was the dominant family in Palapatty (Ganesan et al. 2009) and Sethur sacred groves also (Sambandan \& Dhatchanamoorthy 2012). Ficus is the dominant genus with five species followed by Dioscorea and Solanum comprising three species each; Adiantum, Antidesma, Ardisia, Boehmeria, Bridelia, Clematis, Commelina, Lindernia, Oxalis, Peperomia, Phyllanthus, Piper and Vanda have two species each and 165 genera have single species each. The present investigation on sacred groves reveals that Ficus is dominant genera in Modapalli, these results agreed with the findings of Jamir \& Pandey (2003) and Rao et al. (2010) on Jaintia Hills and Uppa sacred grove of Visakhapatnam district respectively.

A total of 12 species are listed as threatened in this sacred grove along with Ophiorrhiza chandrasekharanii, which is endemic to Visakhapatnam (Rao \& Kumari 2002-2008). Two species viz., Gnetum ula and Lasia spinosa are Least Concern (IUCN 2014); the Endangered species of Cyathea gigantea (Rao et al. 2010) and Lassiococca comberi (Rao et al. 2003) contributed by one and four individuals; some are state level threatened species such as Anodendron paniculatum, Nervilia aragoana, Raphidophora decursiva (one individual each) and Zingeber roseum (two individuals) assessed as Endangered and Oroxylum indicum, Rubia cordifolia, Stemona tuberosa (Reddy \& Reddy 2008) are assessed as Vulnerable (Image $1 \mathrm{a}-\mathrm{n})$. Some of the tall trees like Mangifera indica, Michelia champaka and Elaeocarpus tectorius of height $25 \mathrm{~m}$ are present in the middle of the grove. The most serious threat to the floristic diversity is observed from invasive species, i.e., Lantana camara, Chromolaena odorata and Bidens pilosa causing depletion to the indigenous plant diversity.

\section{CONCLUSION}

This study revealed the presence of a number of plant species along with threatened plants in the sacred grove. This sacred grove has been deteriorating under constant anthropogenic pressure. Natural populations are under serious threat from fire and cultivation of coffee, in and around the sacred grove. Constant grazing, extraction of fuel wood, collection of medicinal plants and non timber forest products causes the degradation of various rare and threatened plants in the sacred grove. This kind of degraded sacred grove should be immediately restored or regenerated using appropriate technologies and by creating awareness about the significance of sacred groves in the maintenance of biodiversity. Therefore, it is important to take appropriate measures and protect such ecologically important groves. The legal status and management of sacred groves in the country needs to 

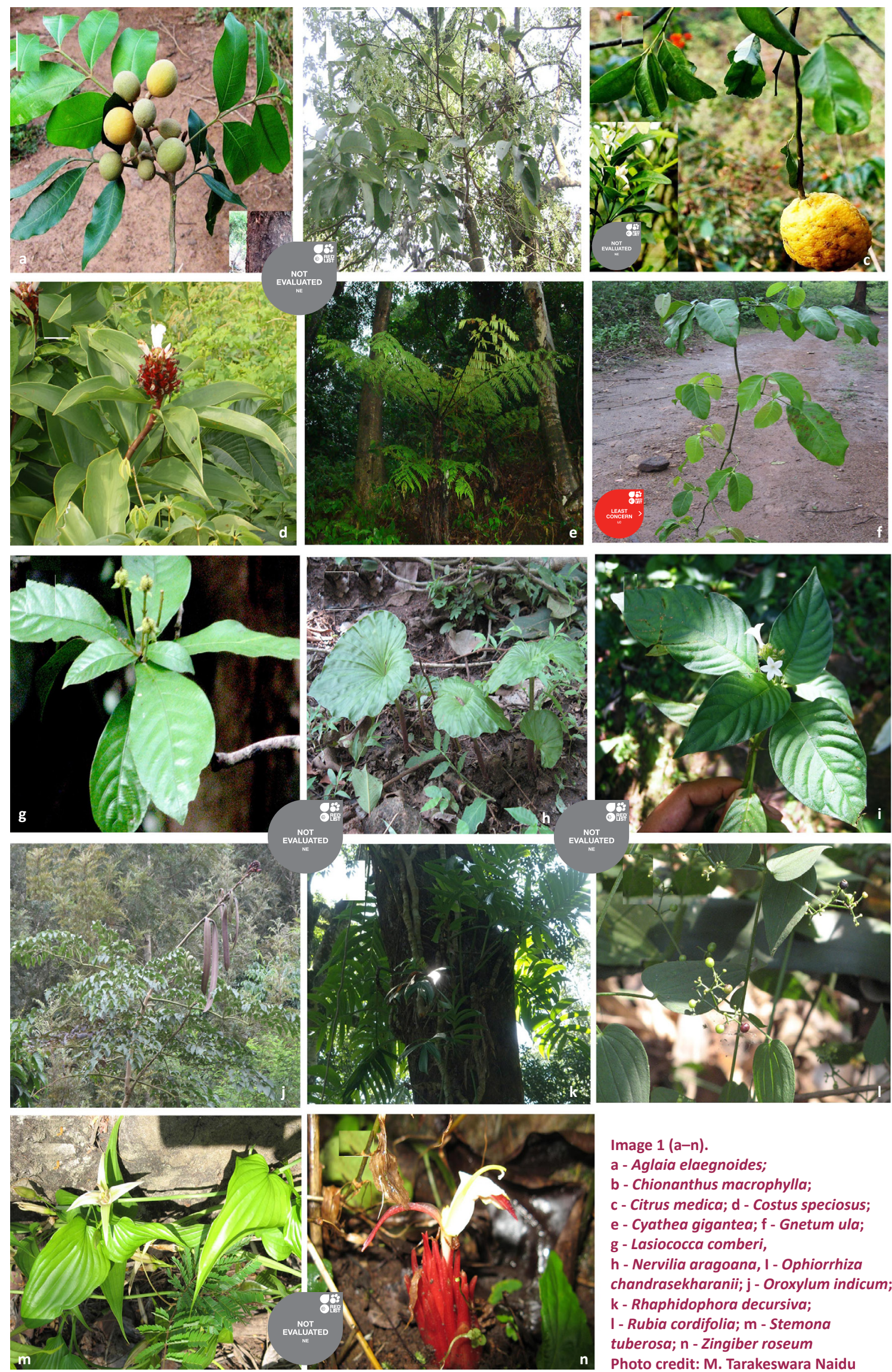

Image 1 (a-n).

a - Aglaia elaegnoides;

- Chionanthus macrophylla;

c - Citrus medica; d - Costus speciosus;

e - Cyathea gigantea; f - Gnetum ula;

g - Lasiococca comberi,

h - Nervilia aragoana, I - Ophiorrhiza

chandrasekharanii; j - Oroxylum indicum;

k - Rhaphidophora decursiva;

I - Rubia cordifolia; m - Stemona

tuberosa; $\mathrm{n}$ - Zingiber roseum

Photo credit: M. Tarakeswara Naidu 
Table 1. List of plant species recorded in the sacred grove of Modapalli.

\begin{tabular}{|c|c|c|c|c|c|c|c|c|c|}
\hline & Species & Habit & Family & $\begin{array}{l}\text { Vernacular } \\
\text { name }\end{array}$ & & Species & Habit & Family & \begin{tabular}{|l} 
Vernacular \\
name
\end{tabular} \\
\hline 1 & $\begin{array}{l}\text { Acalypha ciliata } \\
\text { Forssk. }\end{array}$ & $\mathrm{H}$ & Euphorbiaceae & Merapaku & 32 & $\begin{array}{l}\text { Canthium dicoccum } \\
\text { (Gaertn.) Teijsm. \& }\end{array}$ & $\mathrm{T}$ & Rubiaceae & Alli \\
\hline \multirow{2}{*}{2} & \multirow{2}{*}{$\begin{array}{l}\text { Achyranthes } \\
\text { bidentata } \mathrm{Bl} \text {. }\end{array}$} & \multirow{2}{*}{$\mathrm{H}$} & \multirow{2}{*}{ Amaranthaceae } & & & Binn. & & & \\
\hline & & & & & \multirow{2}{*}{33} & \multirow{2}{*}{$\begin{array}{l}\text { Carmona retusa } \\
\text { (Vahl) Masam. }\end{array}$} & \multirow{2}{*}{ S } & \multirow{2}{*}{ Cordiaceae } & \multirow{2}{*}{ Bapanaburri } \\
\hline \multirow{2}{*}{3} & \multirow{2}{*}{$\begin{array}{l}\text { Adhatoda vasica } \\
\text { Nees }\end{array}$} & \multirow{2}{*}{ S } & \multirow{2}{*}{ Acanthaceae } & \multirow{2}{*}{ Addasaram } & & & & & \\
\hline & & & & & \multirow[b]{2}{*}{34} & \multirow{2}{*}{$\begin{array}{l}\text { Catunaregum } \\
\text { tomentosa (DC.) } \\
\text { Tirv. } \\
\end{array}$} & \multirow[b]{2}{*}{ S } & \multirow[b]{2}{*}{ Rubiaceae } & \multirow[b]{2}{*}{ Kotuvagochh } \\
\hline 4 & $\begin{array}{l}\text { Adiantum caudatum } \\
\text { L. }\end{array}$ & $\mathrm{H}$ & Adiantaceae & Rajahamsa & & & & & \\
\hline 5 & $\begin{array}{l}\text { Adiantum lunulatum } \\
\text { Burm. }\end{array}$ & $\mathrm{H}$ & Adiantaceae & Hamsapadi & 35 & $\begin{array}{l}\text { Cayratia pedata } \\
\text { (Lam.) Gagnep. }\end{array}$ & $\mathrm{Cl}$ & Vitaceae & \\
\hline 6 & $\begin{array}{l}\text { Ageratum } \\
\text { conyzoides L. }\end{array}$ & $\mathrm{H}$ & Asteraceae & & 36 & $\begin{array}{l}\text { Celtis tetrandra } \\
\text { Roxb. }\end{array}$ & $\mathrm{T}$ & Ulmaceae & Jabjabal \\
\hline 7 & $\begin{array}{l}\text { Aglaia elaegnoides } \\
\text { L. }\end{array}$ & $\mathrm{T}$ & Meliaceae & Yerra adugu & 37 & $\begin{array}{l}\text { Centella asiatica (L.) } \\
\text { Urban }\end{array}$ & $\mathrm{H}$ & Apiaceae & Saraswati aku \\
\hline 8 & $\begin{array}{l}\text { Albizia odoratissima } \\
\text { (L.f.) Benth. }\end{array}$ & $\mathrm{T}$ & Mimosaceae & Chinduga & 38 & $\begin{array}{l}\text { Chionanthus } \\
\text { macrophylla Blume }\end{array}$ & $\mathrm{T}$ & Oleaceae & Kurwa \\
\hline 9 & $\begin{array}{l}\text { Alocasia montana } \\
\text { (Roxb.) Schott }\end{array}$ & $\mathrm{H}$ & Araceae & $\begin{array}{l}\text { Bonasaru } \\
\text { Kanda }\end{array}$ & 39 & $\begin{array}{l}\text { Chlorophytum } \\
\text { arundinaceum Baker }\end{array}$ & $\mathrm{H}$ & Liliaceae & Kusala \\
\hline 10 & $\begin{array}{l}\text { Amaranthus viridis } \\
\text { L. }\end{array}$ & $\mathrm{H}$ & Amaranthaceae & Thotakura & 40 & $\begin{array}{l}\text { Chromolaena } \\
\text { odorata (L.) King \& } \\
\text { Robinson }\end{array}$ & S & Asteraceae & Kampurodda \\
\hline 11 & $\begin{array}{l}\text { Anodendron } \\
\text { paniculatum (Roxb) } \\
\text { DC. }\end{array}$ & $\mathrm{Cl}$ & Apocynaceae & & 41 & $\begin{array}{l}\text { Cipadessa baccifera } \\
\text { (Roth.) Miq. }\end{array}$ & s & Meliaceae & Phaladonda \\
\hline \multirow[t]{2}{*}{12} & \multirow{2}{*}{$\begin{array}{l}\text { Antidesma } \\
\text { ghaesembilla } \\
\text { Gaertn. }\end{array}$} & \multirow[t]{2}{*}{$\mathrm{T}$} & \multirow[t]{2}{*}{ Stilaginaceae } & \multirow[t]{2}{*}{ Pulleru } & 42 & $\begin{array}{l}\text { Cissampelos pareira } \\
\text { L. }\end{array}$ & $\mathrm{Cl}$ & Menispermaceae & Tingi teeva \\
\hline & & & & & 43 & Citrus medica L. & $\mathrm{T}$ & Rutaceae & Lungamu \\
\hline 13 & $\begin{array}{l}\text { Antidesma menasu } \\
\text { Tul. }\end{array}$ & $\mathrm{T}$ & Stilaginaceae & & \multirow[t]{2}{*}{44} & \multirow{2}{*}{$\begin{array}{l}\text { Clausena } \\
\text { heptaphylla (Roxb.) } \\
\text { Wt. \& Arn. }\end{array}$} & $\mathrm{s}$ & Rutaceae & Kondakarive- \\
\hline 14 & Ardisia depressa $\mathrm{Cl}$. & $\mathrm{s}$ & Myrsinaceae & Kuna mokka & & & & & \\
\hline 15 & $\begin{array}{l}\text { Ardisia solanacea } \\
\text { Roxb. }\end{array}$ & s & Myrsinaceae & Kuntikaya & 45 & $\begin{array}{l}\text { Cleidion javanicum } \\
\text { Blume }\end{array}$ & $\mathrm{T}$ & Euphorbiaceae & \\
\hline 16 & $\begin{array}{l}\text { Argyreia nervosa } \\
\text { (Burm. f.) Boj. }\end{array}$ & $\mathrm{Cl}$ & Convolvulaceae & Samudrapala & 46 & $\begin{array}{l}\text { Cleistanthus patulus } \\
\text { (Roxb.) Muell.-Arg. }\end{array}$ & $\mathrm{T}$ & Euphorbiaceae & Jiguru \\
\hline 17 & $\begin{array}{l}\text { Arisaema tortuosum } \\
\text { (Wall.) Schott }\end{array}$ & $\mathrm{H}$ & Araceae & Pamu kanda & 47 & $\begin{array}{l}\text { Clematis gouriana } \\
\text { DC }\end{array}$ & $\mathrm{Cl}$ & Ranunculaceae & Gowrikuntala \\
\hline 18 & $\begin{array}{l}\text { Artocarpus lacucha } \\
\text { Buch.-Ham. }\end{array}$ & $\mathrm{T}$ & Moraceae & Nakkarenu & 48 & $\begin{array}{l}\text { Clematis smilacifolia } \\
\text { Wall. }\end{array}$ & $\mathrm{Cl}$ & Ranunculaceae & Budia mal \\
\hline 19 & Arundo donax L. & $\mathrm{Cl}$ & Poaceae & Kakiveduru & 49 & $\begin{array}{l}\text { Clerodendrum } \\
\text { viscosum Vent. }\end{array}$ & S & Verbenaceae & $\begin{array}{l}\text { Bommala- } \\
\text { marri }\end{array}$ \\
\hline 20 & $\begin{array}{l}\text { Atalantia } \\
\text { monophylla (L.) Corr. }\end{array}$ & $\mathrm{s}$ & Rutaceae & Karunimma & 50 & Coffea arabica L. & S & Rubiaceae & Coffee \\
\hline 21 & $\begin{array}{l}\text { Barleria strigosa } \\
\text { Willd. }\end{array}$ & $\mathrm{H}$ & Acanthaceae & & 51 & $\begin{array}{l}\text { Colebrookea } \\
\text { oppositifolia J.E.Sm. }\end{array}$ & S & Lamiaceae & \\
\hline 22 & Bidens pilosa $\mathrm{L}$. & $\mathrm{H}$ & Asteraceae & & 52 & $\begin{array}{l}\text { Colocasia esculenta } \\
\text { (L.) Schott. \& Endl. }\end{array}$ & $\mathrm{H}$ & Araceae & Chamadumpa \\
\hline 23 & $\begin{array}{l}\text { Blechnum orientale } \\
\mathrm{L} .\end{array}$ & $\mathrm{H}$ & Blechnaceae & & 53 & $\begin{array}{l}\text { Commelina } \\
\text { benghalensis } \mathrm{L} \text {. }\end{array}$ & $\mathrm{H}$ & Commelinaceae & Amruthakada \\
\hline 24 & $\begin{array}{l}\text { Blumea mollis } \\
\text { (D.Don) Merr. }\end{array}$ & $\mathrm{H}$ & Asteraceae & & 54 & $\begin{array}{l}\text { Commelina } \\
\text { maculata Edgew. }\end{array}$ & $\mathrm{H}$ & Commelinaceae & \\
\hline 25 & $\begin{array}{l}\text { Boehmaria } \\
\text { glomerulifera L. }\end{array}$ & $\mathrm{H}$ & Urticaceae & & 55 & $\begin{array}{l}\text { Corallocarpus } \\
\text { epigaeus (Rottl. \& }\end{array}$ & $\mathrm{Cl}$ & Cucurbitaceae & Kakidonda \\
\hline & Boehmaria & & & & & Willd.) Cl. & & & \\
\hline 26 & $\begin{array}{l}\text { macrophylla } \\
\text { Hornem. }\end{array}$ & $\mathrm{H}$ & Urticaceae & Keringa & 56 & $\begin{array}{l}\text { Costus speciosus } \\
\text { (Koen.) Sm. }\end{array}$ & $\mathrm{H}$ & Costaceae & Bogachika \\
\hline 27 & $\begin{array}{l}\text { Brachiaria ramosa } \\
\text { (L.) Stapf. }\end{array}$ & $\mathrm{H}$ & Poaceae & Eduru gaddi & 57 & Crinum latifolium L. & $\mathrm{H}$ & Amarylladaceae & \\
\hline 28 & $\begin{array}{l}\text { Bridelia airy-shawii } \\
\text { P.T.Li }\end{array}$ & $\mathrm{T}$ & Euphorbiaceae & Cluytia & 58 & $\begin{array}{l}\text { Curculigo } \\
\text { trichocarpa (Baker) } \\
\text { Hook.f. }\end{array}$ & $\mathrm{H}$ & Hypoxidaceae & Nakkudumpa \\
\hline 29 & $\begin{array}{l}\text { Bridelia pubescens } \\
\text { Kurz }\end{array}$ & $\mathrm{T}$ & Euphorbiaceae & & & Curcuma & & & \\
\hline 30 & $\begin{array}{l}\text { Callicarpa } \\
\text { tomentosa (L.) Murr. }\end{array}$ & $\mathrm{T}$ & Verbenaceae & Sanka Gochi & 59 & $\begin{array}{l}\text { pseudomantana } \\
\text { Graham }\end{array}$ & $\mathrm{H}$ & Zingiberaceae & \\
\hline 31 & $\begin{array}{l}\text { Canscora diffusa } \\
\text { (Vahl) R. Br. }\end{array}$ & $\mathrm{H}$ & Gentianaceae & & 60 & $\begin{array}{l}\text { Cyanotis cristata } \\
\text { (L.) Don }\end{array}$ & $\mathrm{H}$ & Commelinaceae & Eggoli \\
\hline
\end{tabular}




\begin{tabular}{|c|c|c|c|c|c|c|c|c|c|}
\hline & Species & Habit & Family & $\begin{array}{l}\text { Vernacular } \\
\text { name }\end{array}$ & & Species & Habit & Family & $\begin{array}{l}\text { Vernacular } \\
\text { name }\end{array}$ \\
\hline \multirow[t]{2}{*}{61} & \multirow{2}{*}{$\begin{array}{l}\text { Cyathea gigantea } \\
\text { (Wall. ex Hook.) } \\
\text { Holttum }\end{array}$} & \multirow[t]{2}{*}{$\mathrm{T}$} & \multirow[t]{2}{*}{ Cyatheaceae } & \multirow[t]{2}{*}{ Pedda kollem } & 92 & $\begin{array}{l}\text { Ficus semicordata } \\
\text { Sm. }\end{array}$ & $\mathrm{T}$ & Moraceae & Erubodda \\
\hline & & & & & \multirow{2}{*}{93} & \multirow{2}{*}{$\begin{array}{l}\text { Firmiana colorata } \\
\text { (Roxb.) R.Br. }\end{array}$} & \multirow{2}{*}{$\mathrm{T}$} & \multirow{2}{*}{ Sterculiaceae } & \multirow{2}{*}{ Karu boppaja } \\
\hline \multirow{2}{*}{62} & \multirow{2}{*}{$\begin{array}{l}\text { Cymbidium } \\
\text { aloifolium (L.) Sw. }\end{array}$} & \multirow{2}{*}{$\mathrm{H}$} & \multirow{2}{*}{ Orchidaceae } & & & & & & \\
\hline & & & & & \multirow{2}{*}{94} & \multirow{2}{*}{$\begin{array}{l}\text { Gardenia latifolia } \\
\text { Ait. }\end{array}$} & \multirow{2}{*}{$\mathrm{T}$} & \multirow{2}{*}{ Rubiaceae } & \multirow{2}{*}{ Pedda bikki } \\
\hline \multirow{2}{*}{63} & \multirow{2}{*}{$\begin{array}{l}\text { Cynanchum } \\
\text { callialatum Wt. }\end{array}$} & \multirow{2}{*}{$\mathrm{Cl}$} & Asclepiadaceae & & & & & & \\
\hline & & & & & 95 & Garuga pinnata & $T$ & Burseraceae & Garuga \\
\hline 64 & Cynodon dactylon & $\mathrm{H}$ & Poaceae & Gariki & 90 & Roxb. & 1 & Durseraceate & Jar uga \\
\hline 04 & (L.) Pers. & $\Pi$ & Podale & GdrIKI & 96 & Glohba maranting I & $\mathrm{H}$ & 7ingiberaceae & Kalingarastra \\
\hline & Cynoglosum & & & & 90 & $\mathrm{G}$ Gobo maramtina $\mathrm{L}$. & $\mathrm{H}$ & Limginel detare & Gadda \\
\hline 65 & $\begin{array}{l}\text { zeylanicum } \\
\text { (Hornem.) Lehm. }\end{array}$ & $\mathrm{H}$ & Boraginaceae & & 97 & $\begin{array}{l}\text { Glochidion } \\
\text { velutinum } \mathrm{Wt} .\end{array}$ & $\mathrm{T}$ & Euphorbiaceae & \\
\hline 66 & $\begin{array}{l}\text { Cyperus cyperinus } \\
\text { Suring. }\end{array}$ & $\mathrm{H}$ & Cyperaceae & & 98 & Gnetum ula Brongn. & $\mathrm{Cl}$ & Gnetaceae & Lollodu teega \\
\hline 67 & $\begin{array}{l}\text { Cyrtococcum } \\
\text { oxyphyllum (Steud.) }\end{array}$ & $\mathrm{H}$ & Poaceae & & 99 & $\begin{array}{l}\text { Gomphostemma } \\
\text { parviflorum Benth. }\end{array}$ & H & Lamiaceae & \\
\hline & Stapf & & & & 100 & Gynura & $\mathrm{H}$ & Asteraceae & Pottari \\
\hline & Dactyloctenium & & & & & lycopersifolia DC. & & & \\
\hline 68 & $\begin{array}{l}\text { aegyptium (L.) } \\
\text { Beauv. }\end{array}$ & $\mathrm{H}$ & Poaceae & Ganuku gaddi & 101 & $\begin{array}{l}\text { Habenaria } \\
\text { plantaginea Lindl. }\end{array}$ & H & Orchidaceae & \\
\hline 69 & $\begin{array}{l}\text { Dalbergia } \\
\text { lanceolaria L. f. }\end{array}$ & $\mathrm{Cl}$ & Fabaceae & Yerra pachari & 102 & $\begin{array}{l}\text { Haldinia cordifolia } \\
\text { (Roxb.) Ridsd. }\end{array}$ & $\mathrm{T}$ & Rubiaceae & Kamba \\
\hline 70 & $\begin{array}{l}\text { Debregeasia } \\
\text { longifolia (Burm. f.) }\end{array}$ & $\mathrm{T}$ & Urticaceae & Kerangi & 103 & $\begin{array}{l}\text { Heliotropium } \\
\text { bracteatum } \mathrm{R} . \mathrm{Br} .\end{array}$ & H & Boraginaceae & \\
\hline & & & & & 104 & Hemionitis arifolia & $\mathrm{H}$ & Adiantaceae & Rama hanam \\
\hline & & & & & 104 & (Burm.f.) T. Moore & $\mathrm{H}$ & | Adianilacede & 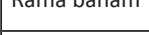 \\
\hline 71 & $\begin{array}{l}\text { aphyllum (Roxb.) } \\
\text { Fisch. }\end{array}$ & $\mathrm{H}$ & Orchidaceae & & 105 & $\begin{array}{l}\text { Homalium } \\
\text { nepaulense Benth. }\end{array}$ & $\mathrm{T}$ & Flacourtiaceae & Tella chettu \\
\hline 72 & $\begin{array}{l}\text { Desmodium } \\
\text { gangeticum (L.) DC. }\end{array}$ & $S$ & Fabaceae & Kolakuponna & 106 & $\begin{array}{l}\text { Homonoia riparia } \\
\text { Lour. }\end{array}$ & S & Euphorbiaceae & \\
\hline 73 & $\begin{array}{l}\text { Dioscorea bulbifera } \\
\text { L. }\end{array}$ & $\mathrm{Cl}$ & Dioscoriaceae & Pendlam & 107 & $\begin{array}{l}\text { Hybanthus } \\
\text { ennaespermus (L.) }\end{array}$ & $\mathrm{H}$ & Violaceae & Ratnapurusa \\
\hline 74 & Dioscorea & $C$ & Dioscoriaceae & Niru Dumna & & F.V. Muell. & & & \\
\hline 14 & oppositifolia L. & C & Diuscuriacear & 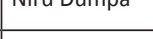 & 108 & Hydrocotyle & $\mathrm{H}$ & Apjaceae & \\
\hline 75 & Dioscorea & $\mathrm{Cl}$ & Dioscoriaceae & & & sibthorpioides Lam. & & & \\
\hline 15 & tomentosa Spreng. & $C_{1}$ & Diuscur lacear & & 109 & Indigofera & $\mathrm{H}$ & Fabaceae & Kondaneeli \\
\hline 76 & Diospyros ovalifolia & $\mathrm{T}$ & Ebenaceae & Kukka Tumiki & & cassioides DC. & & & \\
\hline & Wt. & & & & 110 & Jasminum & $\mathrm{Cl}$ & Oleaceae & Adavi malli \\
\hline 77 & Dolichos trilobus L. & $\mathrm{H}$ & Fabaceae & Pillipesara & & angustifolium Vahl & & & \\
\hline 78 & Drymaria cordata & $\mathrm{H}$ & Carvonbullaceae & & 111 & Kydia calycina Roxb. & $\mathrm{T}$ & Malvaceae & Konda patti \\
\hline & (L.) Roem. \& Schult. & & Caryopnymacede & & 112 & Lantana camara L. & s & Verbenaceae & Gaju pulu \\
\hline 79 & $\begin{array}{l}\text { Duranta plumeri } \\
\text { Jacq. }\end{array}$ & $S$ & Verbenaceae & & 113 & $\begin{array}{l}\text { Laportea crenulata } \\
\text { Gaud. }\end{array}$ & S & Urticaceae & $\begin{array}{l}\text { Yenugu } \\
\text { davadalu }\end{array}$ \\
\hline 80 & $\begin{array}{l}\text { Ecbolium viride } \\
\text { (Forssk.) Alston }\end{array}$ & $\mathrm{H}$ & Acanthaceae & & 114 & $\begin{array}{l}\text { Lasia spinosa (L.) } \\
\text { Thw. }\end{array}$ & $\mathrm{H}$ & Araceae & \\
\hline 81 & $\begin{array}{l}\text { Ehretia acuminata } \\
\text { var. serrata } \mathrm{R} \text {. Br. }\end{array}$ & $\mathrm{T}$ & Cordiaceae & $\begin{array}{l}\text { Bochchuc- } \\
\text { hettu }\end{array}$ & 115 & $\begin{array}{l}\text { Lasiococca comberi } \\
\text { Haine }\end{array}$ & $\mathrm{T}$ & Euphorbiaceae & \\
\hline 82 & $\begin{array}{l}\text { Elaeocarpus } \\
\text { tectorius (Lour) Poir. }\end{array}$ & $\mathrm{T}$ & Elaeocarpaceae & Dippamanu & 116 & $\begin{array}{l}\text { Leea indica (Burm.f.) } \\
\text { Merr. }\end{array}$ & $\mathrm{T}$ & Leeaceae & \\
\hline 83 & $\begin{array}{l}\text { Elephantopus } \\
\text { scaber L. }\end{array}$ & $\mathrm{H}$ & Asteraceae & Hastapadi & 117 & $\begin{array}{l}\text { Lindernia anagallis } \\
\text { (Burm.f.) Pennell }\end{array}$ & $\mathrm{H}$ & Schrophulariaceae & \\
\hline 84 & $\begin{array}{l}\text { Eragrostis atrovirens } \\
\text { (Desf.) Steud. }\end{array}$ & $\mathrm{H}$ & Poaceae & & 118 & $\begin{array}{l}\text { Lindernia antipoda } \\
\text { (L.) Alston }\end{array}$ & $\mathrm{H}$ & Schrophulariaceae & \\
\hline 85 & $\begin{array}{l}\text { Eranthemum } \\
\text { purpurascens Nees }\end{array}$ & $\mathrm{H}$ & Acanthaceae & & 119 & $\begin{array}{l}\text { Lipocarpa chinensis } \\
\text { (Osbeck) Kern. }\end{array}$ & $\mathrm{H}$ & Cyperaceae & \\
\hline 86 & $\begin{array}{l}\text { Erioglosum } \\
\text { rubiginosum BI. }\end{array}$ & $\mathrm{T}$ & Sapindaceae & Ishirasi & 120 & $\begin{array}{l}\text { Luisia zeylanica } \\
\text { Lindl. }\end{array}$ & $\mathrm{H}$ & Orchidaceae & \\
\hline 87 & $\begin{array}{l}\text { Erythrina suberosa } \\
\text { Roxb. }\end{array}$ & $\mathrm{T}$ & Fabaceae & \begin{tabular}{|l} 
Mullu \\
Moduga \\
\end{tabular} & 121 & $\begin{array}{l}\text { Lygodium flexuosum } \\
\text { (L.) Sw. }\end{array}$ & $\mathrm{Cl}$ & Schizaceae & Adavi satavari \\
\hline 89 & $\begin{array}{l}\text { Ficus auriculata } \\
\text { Lour. }\end{array}$ & $\mathrm{T}$ & Moraceae & Racha Bodda & 122 & $\begin{array}{l}\text { Macaranga peltata } \\
\text { (Roxb.) Muell.-Arg. }\end{array}$ & $\mathrm{T}$ & Euphorbiaceae & Konda tamara \\
\hline 88 & Ficus elastic Roxb. & $\mathrm{T}$ & Moraceae & Rubber tree & 123 & Malaxis rheedii Sw. & $\mathrm{H}$ & Orchidaceae & Jivadumpa \\
\hline 90 & Ficus hispida L.f. & T & Moraceae & Bodda & & Mallotus & & & \\
\hline 91 & Ficus microcarpa L.f. & $\mathrm{T}$ & Moraceae & Konda juvvi & 124 & $\begin{array}{l}\text { philippensis (Lam.) } \\
\text { Muell.-Arg. }\end{array}$ & $\mathrm{T}$ & Euphorbiaceae & Sindhuram \\
\hline
\end{tabular}




\begin{tabular}{|c|c|c|c|c|}
\hline & Species & Habit & Family & $\begin{array}{l}\text { Vernacular } \\
\text { name }\end{array}$ \\
\hline 125 & $\begin{array}{l}\text { Malvastrum } \\
\text { coromandelianum } \\
\text { (L.) Garcke }\end{array}$ & H & Malvaceae & \\
\hline 126 & Mangifera indica $\mathrm{L}$. & $\mathrm{T}$ & Anacardiaceae & Mamidi \\
\hline 127 & $\begin{array}{l}\text { Manilkara hexandra } \\
\text { (Roxb.) Dubard. }\end{array}$ & $\mathrm{T}$ & Sapotaceae & Pala Chettu \\
\hline 128 & $\begin{array}{l}\text { Maytenus hookeri } \\
\text { Loes. }\end{array}$ & $\mathrm{T}$ & Celastraceae & \\
\hline 129 & $\begin{array}{l}\text { Melastoma } \\
\text { malabathricum L. }\end{array}$ & $S$ & Melastomataceae & Niti danimma \\
\hline 130 & $\begin{array}{l}\text { Michelia champaca } \\
\text { L. }\end{array}$ & $\mathrm{T}$ & Magnoliaceae & Sampangi \\
\hline 131 & $\begin{array}{l}\text { Micrococca } \\
\text { mercurialis (L.) } \\
\text { Benth }\end{array}$ & H & Euphorbiaceae & \\
\hline 132 & $\begin{array}{l}\text { Millettia racemosa } \\
\text { (Wt. \& Arn.) Benth }\end{array}$ & $\mathrm{Cl}$ & Fabaceae & Kudai goch \\
\hline 133 & Mimosa pudica $\mathrm{L}$. & $\mathrm{H}$ & Mimosaceae & Attipatti \\
\hline 134 & $\begin{array}{l}\text { Murdannia nudiflora } \\
\text { (L.) Brenan }\end{array}$ & $\mathrm{H}$ & Commelinaceae & \\
\hline 135 & $\begin{array}{l}\text { Murraya paniculata } \\
\text { (L.) Jack. }\end{array}$ & $S$ & Rutaceae & Puvelaga \\
\hline 136 & Musa rosacea Jacq. & H & Musaceae & Konda arati \\
\hline 137 & $\begin{array}{l}\text { Naringi crenulata } \\
\text { (Roxb.) Nicols. }\end{array}$ & T & Rutaceae & Torruvelaga \\
\hline 138 & $\begin{array}{l}\text { Neolitsea cassia (L.) } \\
\text { Kosterm. }\end{array}$ & $\mathrm{T}$ & Lauraceae & Mori \\
\hline 139 & $\begin{array}{l}\text { Nervilia aragoana } \\
\text { Gaud. }\end{array}$ & H & Orchidaceae & \begin{tabular}{|l} 
Ontelud- \\
umpa
\end{tabular} \\
\hline 140 & $\begin{array}{l}\text { Nervilia plicata } \\
\text { (Andr.) Schltr. }\end{array}$ & H & Orchidaceae & \\
\hline 141 & $\begin{array}{l}\text { Ophiorrhiza } \\
\text { chandrasekharanii } \\
\text { Subba Rao \& Kumari }\end{array}$ & H & Rubiaceae & \\
\hline 142 & $\begin{array}{l}\text { Oplisminus burmanii } \\
\text { (Retz.) Beauv. }\end{array}$ & $\mathrm{H}$ & Poaceae & $\begin{array}{l}\text { Kodijuttu } \\
\text { gaddi }\end{array}$ \\
\hline 143 & $\begin{array}{l}\text { Oroxylum indicum } \\
\text { (L.) Vent. }\end{array}$ & $\mathrm{T}$ & Bignoniaceae & Pampini \\
\hline 144 & $\begin{array}{l}\text { Orthosiphon } \\
\text { rubicundus (D. Don) } \\
\text { Benth. }\end{array}$ & $\mathrm{H}$ & Lamiaceae & Poda tulasi \\
\hline 145 & Oxalis corniculata L. & $\mathrm{H}$ & Oxalidaceae & Pulichinta \\
\hline 146 & Oxalis latifolia HBK & $\mathrm{H}$ & Oxalidaceae & \\
\hline 147 & $\begin{array}{l}\text { Parabaena sagittata } \\
\text { Miers }\end{array}$ & $\mathrm{Cl}$ & Menispermaceae & \\
\hline 148 & $\begin{array}{l}\text { Pennisetum } \\
\text { pedicellatum Trin. }\end{array}$ & $\mathrm{H}$ & Poaceae & \\
\hline 149 & $\begin{array}{l}\text { Peperomia } \\
\text { dindigulensis Miq. }\end{array}$ & $\mathrm{H}$ & Piperaceae & \\
\hline 150 & $\begin{array}{l}\text { Peperomoa } \\
\text { tetraphylla (Forst.f.) } \\
\text { Hook. \& Arn. }\end{array}$ & $\mathrm{H}$ & Piperaceae & \\
\hline 151 & $\begin{array}{l}\text { Phoebe wightii } \\
\text { Meissn. }\end{array}$ & $\mathrm{T}$ & Lauraceae & \\
\hline 152 & $\begin{array}{l}\text { Phyllanthus airy- } \\
\text { shawii Jean F.Brunel } \\
\text { \& J.P.Roux }\end{array}$ & $\mathrm{H}$ & Euphorbiaceae & \\
\hline 153 & $\begin{array}{l}\text { Phyllanthus emblica } \\
\text { L. }\end{array}$ & $\mathrm{T}$ & Euphorbiaceae & Usiri \\
\hline 154 & $\begin{array}{l}\text { Pilea melastomoides } \\
\text { (Poir.) Wedd. }\end{array}$ & $\mathrm{H}$ & Urticaceae & Dongorbhori \\
\hline 155 & Piper longum L. & $\mathrm{Cl}$ & Piperaceae & Pippallu \\
\hline 156 & Piper trioicum Roxb. & $\mathrm{Cl}$ & Piperaceae & Mirialu \\
\hline
\end{tabular}

\begin{tabular}{|c|c|c|c|c|}
\hline & Species & Habit & Family & $\begin{array}{l}\text { Vernacular } \\
\text { name }\end{array}$ \\
\hline 157 & $\begin{array}{l}\text { Plectranthus mollis } \\
\text { (Ait.) Spreng. }\end{array}$ & $\mathrm{H}$ & Lamiaceae & \\
\hline 158 & $\begin{array}{l}\text { Pleopeltis } \\
\text { macrocarpa } \\
\text { (Bory ex Willd.) } \\
\text { Kaulf. }\end{array}$ & $\mathrm{H}$ & Polypodiaceae & \\
\hline 159 & $\begin{array}{l}\text { Pogostemon } \\
\text { benghalensis } \\
\text { (Burm.f.) O. Ktze. }\end{array}$ & $\mathrm{H}$ & Lamiaceae & Dumbadal \\
\hline 160 & $\begin{array}{l}\text { Polygonum } \\
\text { barbatum var. } \\
\text { gracile } \mathrm{L} .\end{array}$ & $\mathrm{H}$ & Polygonaceae & \\
\hline 161 & $\begin{array}{l}\text { Polygonum chinense } \\
\text { L. }\end{array}$ & $\mathrm{H}$ & Polygonaceae & Kuna mokka \\
\hline 162 & $\begin{array}{l}\text { Protium serratum } \\
\text { (Cilebr.) Engl. }\end{array}$ & $\mathrm{T}$ & Burseraceae & Busi \\
\hline 163 & Psidium gaujava L. & $\mathrm{T}$ & Myrtaceae & Jama \\
\hline 164 & $\begin{array}{l}\text { Psychotria } \\
\text { monticola Kurz. }\end{array}$ & H & Rubiaceae & \\
\hline 165 & Pteris vittata & $\mathrm{H}$ & Pteridaceae & \\
\hline 166 & $\begin{array}{l}\text { Pterocarpus } \\
\text { marsupium Roxb. }\end{array}$ & $\mathrm{T}$ & Fabaceae & Yegisa \\
\hline 167 & $\begin{array}{l}\text { Remusatia vivipara } \\
\text { (Roxb.) Schott. }\end{array}$ & $\mathrm{H}$ & Araceae & \\
\hline 168 & $\begin{array}{l}\text { Rhaphidophora } \\
\text { decursiva (Roxb.) } \\
\text { Schott. }\end{array}$ & $\mathrm{Cl}$ & Araceae & $\begin{array}{l}\text { Athukula } \\
\text { chettu }\end{array}$ \\
\hline 169 & $\begin{array}{l}\text { Rhopalephora } \\
\text { scaberrima (Bl.) } \\
\text { Faden }\end{array}$ & $\mathrm{H}$ & Commelinaceae & \\
\hline 170 & $\begin{array}{l}\text { Rhynchostylis retusa } \\
\text { (L.) Bl. }\end{array}$ & $\mathrm{H}$ & Orchidaceae & \\
\hline 171 & $\begin{array}{l}\text { Rotala densiflora } \\
\text { (Roem. \& Schult.) } \\
\text { Koehne }\end{array}$ & $\mathrm{H}$ & Lythraceae & \\
\hline 172 & Rubia cordifolia L. & $\mathrm{H}$ & Rubiaceae & Mangala katti \\
\hline 173 & Rubus ellipticus Sm. & S & Rosaceae & Gedapallu \\
\hline 174 & $\begin{array}{l}\text { Sapium } \\
\text { eugeniifolium } \\
\text { Hook.f. }\end{array}$ & $\mathrm{T}$ & Euphorbiaceae & \\
\hline 175 & $\begin{array}{l}\text { Schefflera stellata } \\
\text { (Gaertn.) Roxb. }\end{array}$ & $\mathrm{Cl}$ & Araliaceae & Reval \\
\hline 176 & $\begin{array}{l}\text { Schleichera oleosa } \\
\text { (Lour) Oken }\end{array}$ & $\mathrm{T}$ & Sapindaceae & Karaka busi \\
\hline 177 & $\begin{array}{l}\text { Selaginella } \\
\text { involvense (Swartz) } \\
\text { Spring }\end{array}$ & $\mathrm{H}$ & Selaginellaceae & Sanjivani \\
\hline 178 & $\begin{array}{l}\text { Setaria pumila } \\
\text { (Poir.) Roem. } \\
\text { \&Schult. } \\
\end{array}$ & $\mathrm{H}$ & Poaceae & $\begin{array}{l}\text { Nakkatoka } \\
\text { gaddi }\end{array}$ \\
\hline 179 & Sida acuta Burm.f. & $\mathrm{H}$ & Malvaceae & Polikampa \\
\hline 180 & $\begin{array}{l}\text { Sloanea sterculiacea } \\
\text { (Benth.) Rehder \& } \\
\text { Wilson } \\
\end{array}$ & $\mathrm{T}$ & Sterculiaceae & \\
\hline 181 & Smilax zeylanica L. & $\mathrm{Cl}$ & Smilacaceae & Kummari tivva \\
\hline 182 & $\begin{array}{l}\text { Solanum erianthum } \\
\text { D. Don }\end{array}$ & S & Solanaceae & \\
\hline 183 & $\begin{array}{l}\text { Solanum giganteum } \\
\text { Jacq. }\end{array}$ & S & Solanaceae & \\
\hline 184 & Solanum nigrum $\mathrm{L}$. & $\mathrm{H}$ & Solanaceae & Kamanchi \\
\hline 185 & $\begin{array}{l}\text { Sorghum halepense } \\
\text { (L.) Pers. }\end{array}$ & $\mathrm{H}$ & Poaceae & Gaddi jonna \\
\hline 186 & $\begin{array}{l}\text { Spermadictyon } \\
\text { suaveolens Roxb. }\end{array}$ & $\mathrm{T}$ & Rubiaceae & \\
\hline
\end{tabular}




\begin{tabular}{|c|c|c|c|c|}
\hline & Species & Habit & Family & $\begin{array}{l}\text { Vernacular } \\
\text { name }\end{array}$ \\
\hline 187 & $\begin{array}{l}\text { Spilanthes } \\
\text { paniculata DC. }\end{array}$ & H & Asteraceae & $\begin{array}{l}\text { Pippannu } \\
\text { puvvu }\end{array}$ \\
\hline 188 & $\begin{array}{l}\text { Stachytarpheta } \\
\text { urticaefolia (Salisb.) } \\
\text { Sims }\end{array}$ & $\mathrm{H}$ & Verbenaceae & \\
\hline 189 & $\begin{array}{l}\text { Stemona tuberosa } \\
\text { Lour. }\end{array}$ & $\mathrm{H}$ & Stemanaceae & Adavi tamara \\
\hline 190 & $\begin{array}{l}\text { Synadenium grantii } \\
\text { Hook.f. }\end{array}$ & S & Euphorbiaceae & \\
\hline 191 & $\begin{array}{l}\text { Synedrella nodiflora } \\
\text { (L.) Gaertn. }\end{array}$ & $\mathrm{H}$ & Asteraceae & \\
\hline 192 & $\begin{array}{l}\text { Talinum } \\
\text { portulacifolium } \\
\text { (Forsk.) Asch. \& } \\
\text { Schweinf. }\end{array}$ & $\mathrm{H}$ & Portulacaceae & $\begin{array}{l}\text { Ceylone } \\
\text { bachali }\end{array}$ \\
\hline 193 & $\begin{array}{l}\text { Terminalia bellerica } \\
\text { (Gaertn.) Roxb. }\end{array}$ & $\mathrm{T}$ & Combretaceae & Tani \\
\hline 194 & $\begin{array}{l}\text { Tetrastigma } \\
\text { lanceolarium (Roxb.) } \\
\text { Planch. }\end{array}$ & $\mathrm{Cl}$ & Vitaceae & $\begin{array}{l}\text { Gummadi- } \\
\text { baddu }\end{array}$ \\
\hline 195 & $\begin{array}{l}\text { Thalictrum } \\
\text { foliolosum DC. }\end{array}$ & $\mathrm{H}$ & Ranunculaceae & \\
\hline
\end{tabular}

Habit: Cl - Climber; H - Herb; S - Shrub; T - Tree

be examined and there is an urgent need to preserve and acknowledge the efforts of the people of this area in preserving other sacred patches of forest as important areas of local biodiversity.

\section{REFERENCES}

Anthwal, A., R.C. Sharma \& A. Sharma (2006). Sacred groves: traditional way of conserving plant diversity in Garhwal Himalaya, Uttaranchal. Journal of American Science 2(2): 35-38.

Champion, H.G. \& S.K. Seth (1968). Revised survey of forest types of India. Government of India Press, Delhi, India, 117pp.

Chandrashekara, U.M. \& S. Sankar (1998). Ecology and management of sacred grove in Kerala, India. Forest Ecology and Management 112: 165-177; http://dx.doi.org/10.1016/s0378-1127(98)00326-0

Gadgil, M. \& V.D. Vartak (1975). Sacred groves of India: a plea for continued conservation. Journal of the Bombay Natural History Society 72: 314-320

Gamble, J.S. \& C.E.C. Fischer (1915-1936). Flora of the Presidency of Madras 3 Volumes. London, Rep. ed. 1957. Calcutta.

Ganesan, S., M. Ponnuchamy, L. Kesavan \& A. Selvaraj (2009). Floristic composition and practices on the selected sacred groves of Palapatty Village (Reserved forest), Tamil Nadu. Indian Journal of Traditional Knowledge 8(2): 154-162.

Hooker, J.D. (1872-1897). The flora of British India, 7 Vol. L. Reeve \& Co., London, 425-842pp.

IUCN (2014). IUCN Red List of Threatened Species. Version 2014.1. $<$ www.iucnredlist.org>. Downloaded on 17 June 2014.

Jadhav, S.N. \& K.N. Reddy (2006). Threatened medicinal plants of Andhra Pradesh. ENVIS-SDNP special issue, 18-28pp.

Jain, S.K \& R.R. Rao (1977). A Handbook of Field and Herbarium Methods. Today and Tomorrow printers, BSI, Calcutta, 157pp.

Jamir, S.A. \& H.N. Pandey (2003). Vascular plant diversity in the sacred groves of Jaintia hills in northeast India. Biodiversity and Conservation 12: $1497-1510$

Khullar, P. (1992). Conservation of biodiversity in natural forest through preservation plots-A historical perspective. Indian forester 118(5): 337

\begin{tabular}{|c|c|c|c|c|}
\hline & Species & Habit & Family & $\begin{array}{l}\text { Vernacular } \\
\text { name }\end{array}$ \\
\hline 196 & $\begin{array}{l}\text { Thunbergia fragrans } \\
\text { Roxb. }\end{array}$ & $\mathrm{Cl}$ & Acanthaceae & \\
\hline 197 & $\begin{array}{l}\text { Thysanolaena } \\
\text { maxima Nees }\end{array}$ & $\mathrm{H}$ & Poaceae & Konda chipuru \\
\hline 198 & Toona cilata Roem. & $\mathrm{T}$ & Meliaceae & Gali manu \\
\hline 199 & $\begin{array}{l}\text { Uncaria } \\
\text { sessilifructus Roxb. }\end{array}$ & $\mathrm{Cl}$ & Rubiaceae & \\
\hline 200 & $\begin{array}{l}\text { Vanda tessellata } \\
\text { (Roxb.) Don }\end{array}$ & $\mathrm{H}$ & Orchidaceae & Vega badanika \\
\hline 201 & $\begin{array}{l}\text { Vanda testacea } \\
\text { (Lindl.) Reichb.f. }\end{array}$ & $\mathrm{H}$ & Orchidaceae & \\
\hline 202 & $\begin{array}{l}\text { Viola betonicifolia } \\
\text { J.E. Sm. }\end{array}$ & $\mathrm{H}$ & Violaceae & \\
\hline 203 & Vitex leucoxylon L.f. & $\mathrm{T}$ & Verbenaceae & Konda vavili \\
\hline 204 & $\begin{array}{l}\text { Walsura trifoliata } \\
\text { (A. Juss.) }\end{array}$ & $\mathrm{T}$ & Meliaceae & Yerra valudu \\
\hline 205 & $\begin{array}{l}\text { Xylia xylocarpa } \\
\text { (Roxb.) Taub. }\end{array}$ & $\mathrm{T}$ & Mimosaceae & \begin{tabular}{|l} 
Konda \\
Tangedu
\end{tabular} \\
\hline 206 & $\begin{array}{l}\text { Zanthoxylum } \\
\text { armatum DC. }\end{array}$ & $\mathrm{T}$ & Rutaceae & Tumburu \\
\hline 207 & $\begin{array}{l}\text { Zingiber roseum } \\
\text { (Roxb.) Roscoe }\end{array}$ & $\mathrm{H}$ & Zingiberaceae & Rajuladumpa \\
\hline
\end{tabular}

Lakshminarayana, K. \& M. Venkaiah (1998). Biodiversity in the sacred groves of the north coastal districts of Andhra Pradesh. National symposium on conservation of Eastern Ghats, 52-58pp.

Pullaiah, T \& S. Kuruppusamy (2008). Flora of Andhra Pradesh - Vol. 5. Scientific Publishers, New Delhi, 83pp.

Rao, B.R.P., M.V.S. Babu, M.S. Reddy, V.S. Rao, S. Sunitha \& K.N. Ganesaiah (2011). Sacred groves in sothern Eastern Ghats, India: Are they better managed than forest reserves? Tropical Ecology 52(1): 79-90.

Rao, J.P., M.T. Naidu, P.P. Murty \& M. Venkaiah (2010). Diversity of vascular plants in the sacred grove of Uppa from Eastern Ghats, Visakhapatnam District in Andhra Pradesh. Journal of the Indian Botanical Society 89(3\&4): 406-411.

Rao, K.C., B.L. Geetha \& S. Geetha (2003). Red List of Threatened Vascular Plant Species in India. Botanical Survey of India, Howrah, 43pp.

Rao, G.V.S \& G.R. Kumari (2002-2008). Flora of Visakhapatnam District 2 Volumes. Botanical Survey of India, Kolkata, 612pp.

Rao, P. (1996). Sacred groves and conservation. WWF India (Quart.) 7: 4-7.

Rawat, M., H.B. Vasista, R.K. Manhas \& M. Negi (2011). Sacred forest of Kunjapuri Siddhapeet, Uttarakhand, India. Tropical Ecology 52(2): 219-211.

Reddy, K.N. \& C.S. Reddy (2008). First red list of medicinal plants of Andhra Pradesh, India-conservation assessment and management planning. Ethnobotanical leaflets 12: 103-107.

Sambandan, K. \& N. Dhatchanamoorthy (2012). Studies on the phytodiversity of a sacred grove and its traditional uses in Karaikal District, U.T. Puducherry. Journal of Phytology 4(2): 16-21.

Sujana, K.A. \& C. Sivaperuman (2008). Status and Conservation of threatened flora in selected sacred groves of Coastal Kerala. Eco News 14(2): 6-10.

Sunitha, S. \& B.R.P. Rao (1999). Sacred groves in Kurnool district, Andhra Pradesh, pp. 367-373. In: Sivadasan, M. \& P. Mathew (eds.) Biodiversity, Taxonomy and Conservation of Flowering Plants. Mentor Publishers, Calicut.

Venkaiah, M. (2004). Studies on Vegetation and Flora of Vizianagaram District, Andhra Pradesh. Andhra University Press, Visakhapatnam, 214pp. 